\title{
Clinical Outcome After Rectal Replacement With Side-to-End, Colon-J-Pouch, or Straight Colorectal Anastomosis Following Total Mesorectal Excision
}

\author{
A Swiss Prospective, Randomized, Multicenter Trial (SAKK 40/04)
}

\author{
Walter R. Marti, MD, ${ }^{*}$ Gaudenz Curti, MD, ${ }^{*}$ Heinz Wehrli, MD, $\dagger$ Felix Grieder, MD, $\ddagger$ Michael Graf, MD, $\S$ \\ Beat Gloor, MD, Markus Zuber, MD, || Nicolas Demartines, MD, ${ }^{* *}$ Fabrizio Fasolini, MD, $\dagger \dagger$ \\ Bruno Lerf, MD, \\ Martin Bigler, PhD, SAKK, $\uparrow$ Stefanie Hayoz, PhD, SAKK, $\uparrow$ Karin Ribi, PhD, IBCSG, $\uparrow$ and \\ Christian Hamel, MD||||, the Swiss Group for Clinical Cancer Research (SAKK), Section Surgery
}

\begin{abstract}
Objective: To compare, in a phase 3, prospective, randomized, multi-center clinical trial functional outcome of reconstruction procedures following total mesorectal excision (TME).

Summary Background Data: Intestinal continuity reconstruction following TME is accompanied by postoperative defecation dysfunctions known as "anterior resection syndrome." Commonly used reconstruction techniques are straight colorectal anastomosis (SCA), colon J -pouch (CJP), and side-toend anastomosis (SEA). Comparison of their functional outcomes in prospective, randomized, multi-center studies, including long-term assessments, is lacking.

Methods: Patients requiring TME for histologically proven rectal tumor, with or without neoadjuvant treatment, age $\geq 18$ years, normal sphincter function without history of incontinence, any pretreatment staging or adenoma, expected R0-resection, were randomized for standardized SCA, CJP, or SEA procedures. Primary endpoint was comparison of composite evacuation scores 12 months after TME. Comparison of composite evacuation and incontinence scores at 6,18 and 24 months after surgery, morbidity, and overall survival represented secondary endpoints. Analysis was based on "per protocol" (PP) population, fully complying with trial requirements, and intention-to treat (ITT) population.
\end{abstract}

Results: Three hundred thirty-six patients from 15 hospitals were randomized. $\mathrm{PP}$ population included 257 patients $(\mathrm{JCP}=63 ; \mathrm{SEA}=95 ; \mathrm{SCA}=99)$.

From the *Department of Visceral Surgery, Kantonsspital Aarau, Aarau, Switzerland; †Department of Visceral Surgery, Hirslandenklinik, Zürich, Switzerland; $\ddagger$ Department of Visceral Surgery, Kantonsspital Winterthur, Winterthur, Switzerland; §Department of Visceral Surgery, Kantonsspital Luzern, Luzern, Switzerland; 『Universitätslinik für Viszerale Chirurgie, Inselspital, Bern, Switzerland; ||Department of Surgery, Kantonsspital Olten, Switzerland; **Service de Chirurgie Viscérale, CHUV, Lausanne, Switzerland;

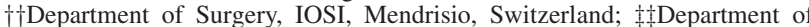
General and Visceral Surgery, Kantonspital Zug, Switzerland; §§Clinic of General Surgery, Basel University Hospital, Basel, Switzerland; - Coordinating Center, Bern, Switzerland; and ||||Clinic for General and Visceral Surgery, Regional Hospital Lörrach, Lörrach, Germany.

This work was partially supported by a grant from Oncosuisse (OCS 01579-082004) and by the Swiss State Secretariat for Education, Research and Innovation (SERI)

The authors report no conflicts of interest.

Supplemental digital content is available for this article. Direct URL citations appear in the printed text and are provided in the HTML and PDF versions of this article on the journal's Web site (www.annalsofsurgery.com).

Reprints: Walter R. Marti, MD, Prof Dr. med., chirurgieaarau, Bahnhofstrasse 24, 5000 Aarau, Switzerland. E-mail: Walter.R.Marti@hin.ch.

Copyright (C) 2018 Wolters Kluwer Health, Inc. All rights reserved.

ISSN: 0003-4932/16/XXXX-0001

DOI: $10.1097 /$ SLA.0000000000003057
Composite evacuation scores of PP and ITT populations did not show statistically significant differences among the 3 groups at any time point. Similarly, composite incontinence scores for PP and ITT populations showed no statistically significant difference among the 3 trial arms at any time point. Conclusions: Within boundaries of investigated procedures, surgeons in charge may continue to perform reconstruction of intestinal continuity following TME at their technical preference.

Keywords: colon J-pouch, colorectal reconstruction, evacuation score, incontinence score, Mesorectal excision, side-to-end anastomosis, straight colorectal anastomosis, straight colorectal anastomosis

(Ann Surg 2018;xx:xxx-xxx)

S urgical resection represents the mainstay of treatment for rectal $\checkmark$ tumors. Thanks to surgical technical progress, in a majority of cases the sphincter complex can be spared and a rectal reconstruction can be performed. However, despite successful reconstruction of intestinal continuity, normal functions are not fully restored, and postoperative symptoms, including fecal incontinence, urgency, and high frequency of defecation, have been described as "anterior resection syndrome.",1,2

To improve functional outcome of straight colorectal anastomosis (SCA), a variety of procedures have been proposed. In particular, Lazorthes and Parc introduced the colon J-pouch (CJP) approach. ${ }^{3,4}$ However, due to defecatory dysfunctions, this form of reconstruction has largely been updated by substantially shortening $\mathbf{J}$ pouches. Side-to-end anastomosis (SEA) $)^{5}$ has also been used to form even smaller reservoirs, thereby combining advantages of the $5 \mathrm{~cm}$ CJP, namely safer anastomosis and lower stool frequencies, with those of SCA, including better evacuation and lesser fragmentation. Although additional techniques, such as ileocoecal interposition ${ }^{6}$ and transverse coloplasty ${ }^{7}$ have also been developed, technical complexity, safety reasons, and debated functional outcome have prevented their widespread adoption.

Randomized, controlled trials comparatively investigating functional outcomes of different techniques have indeed been conducted. ${ }^{8}$ However, studies comparing most frequently used procedures, SEA, CJP and SCA, are generally characterized by limited statistical power due to relatively small numbers of patients included. Furthermore, they frequently represent single-center experiences or report short-term results only. ${ }^{9-16}$

To fill this knowledge gap, in a multi-institutional, randomized, controlled trial, we have compared long-term functional 
outcomes of $5 \mathrm{~cm}$ CJP and SEA with SCA reconstruction following TME.

\section{METHODS}

\section{Trial Design and Participants}

This phase 3, prospective, randomized trial was performed in collaboration by a group of 15 Swiss hospitals, including 4 University hospitals, 3 central hospitals, and 8 regional hospitals. On the basis of the different reconstruction techniques under investigation, the trial design consisted of 3 arms: $5 \mathrm{~cm}$ CJP, SEA, and SCA.

Eligibility criteria included: histologically proven rectal adenocarcinoma or rectal adenoma with or without neoadjuvant radiotherapy or radio-chemotherapy; TME requirement; age $\geq 18$ years; clinically normal function of sphincter muscles with no history of frequent fecal incontinence for liquid or solid stools; pretreatment staging of any T, N, M or adenoma; expected R0-resection whereas resectable synchronous metastases did not lead to exclusion; completed baseline interview on evacuation and incontinence.

Exclusion criteria were: rectal cancer surgery other than local excision in the previous 2 months; planned adjuvant postoperative radiotherapy; histologically proven chronic inflammatory bowel disease; contraindication for any of the surgical reconstruction techniques under investigation; a BMI $>35 \mathrm{~kg} / \mathrm{m}^{2}$; psychiatric, addictive or any disorder prohibiting giving informed consent; inability to read and understand any of the languages available on questionnaires and spoken during the interviews (German, French, Italian).

The full Protocol is available in supplementary materials.

\section{Procedures}

Following standardized tumor staging, patients were either operated as soon as possible or, preferably, 1 week after short course radiotherapy, or 4 to 10 weeks after completion of neoadjuvant treatment, according to international guidelines. ${ }^{17}$

TME had to be performed according to Heald et $\mathrm{al}^{18}$ in an open surgical approach or laparoscopically, according to discretion and experience of individual surgeon in charge of the intervention.

The 3 types of reconstruction had to be performed in a standardized way by surgeons experienced in all 3 reconstruction techniques. To minimize technical variations, a detailed description of the 3 types of reconstruction was provided. As an example, in the case of CJP, length of longitudinal stabling should not exceed $5 \mathrm{~cm}$. For SEA, the blind end of the neorectum should be $4 \mathrm{~cm}$ long. Anastomosis had to be performed either using a circular stapling devise by double stapler technique, or transanally, by pull through technique by hand, using an interrupted suture. At discretion of the surgeon in charge, a temporary ileostomy could be constructed for reconstruction protection. If the randomized form of reconstruction was not feasible for safety reasons, the surgeon in charge was allowed to choose one of the 2 remaining reconstruction techniques.

\section{OUTCOMES}

Primary objective was to compare $5 \mathrm{~cm}$ CJP, SEA, and SCA reconstruction techniques following TME, regarding defecation quality and evacuation problems. Therefore, the composite evacuation score 12 months after TME was defined as primary endpoint of the study.

Secondary objectives were the comparison of the 3 reconstruction techniques regarding composite evacuation scores at 6,18 , and 24 months after surgery; composite incontinence scores, as assessed at the same time points; morbidity; overall survival and rate of noncompliance with randomization.
Baseline functional assessments were derived from a structured interview led by clinicians in charge. Following surgery, functional outcomes were assessed at $6,12,18$, and 24 months by trained study nurses, members of the SAKK Coordinating Center, by structured phone interviews. To allow unbiased, standardized evaluation of anorectal function, they were independent of surgeons or surgical institutions involved in the trial. Questionnaires were sent to patients 1 week before telephone interviews to facilitate conversation and familiarize patients with questions. Considering that anterior resection syndrome symptoms could be characterized by day-to-day variations, patients were asked to provide answers best describing their daily life. ${ }^{19}$ Overall, 9 questionnaires $(<1 \%)$ could not be filled by patients, since they were unable to give the required information. In these cases, follow-up forms were sent to the general practitioner for completion.

\section{Composite Evacuation Score}

Functional outcome regarding evacuation ability was evaluated according to published assessment and scoring instruments. ${ }^{20}$ They analyze different functional aspects, such as use of medication to evacuate, difficulties to empty, digitation to evacuate, return to evacuate, feeling of incomplete evacuation, straining to evacuate, and time needed to evacuate. Patients were asked to subjectively evaluate each of these aspects per given response categories, resulting in 7 subscores, each between 0 and 3 .

If all 7 questions were answered, the composite evacuation score was the sum of the seven subscores, yielding a total between 0 and 21 , with lower scores associated to better function. If 6 of 7 questions were answered, composite evacuation score was calculated as $7 / 6$ times the sum of the 6 available subscores. Scores for patients with 5 or less answered questions were not calculated.

\section{Composite Incontinence Score}

Functional outcome regarding continence was assessed by a similar composite incontinence score, evaluating different aspects of incontinence, including warning before passing motion, ability to differentiate gas from feces, ability to defer evacuation, wearing a pad during the day, wearing a pad at night, incontinence of gas, incontinence of loose stool, incontinence of feces. ${ }^{8}$ Summary score ranges from 0 to 24 with lower scores associated with better function.

\section{Randomization and Masking}

Patients were allocated by using the minimization method and were stratified based on enrolling surgical clinic, patient's gender, distance of the distal tumor margin from the dentate line $(>5 \mathrm{~cm}$ vs. $\leq 5 \mathrm{~cm}$ ), age ( $<70$ vs. $\geq 70$ years), neoadjuvant treatment (no/yes), and distant metastatic disease (M0 vs. M1).

Staff SAKK members in charge of telephone interviews of enrolled patients 6 to 24 months after surgery, were independent of surgeons, blinded to the selected reconstruction method, and were located at the SAKK Coordinating Center in Bern, with no attachments to hospitals involved in the study.

\section{Oncologic Outcome, Morbidity, and Mortality}

Participating surgical centers had to fill specific assessment forms. Short-term, 30-day, morbidity evaluation focused on septic problems, anastomotic leakage and reoperation. Regarding longterm morbidity, formation of anastomotic stenosis or perianal fistula, and non-closure of protective stoma were assessed until the end of the study period (24 months). Overall and disease specific survival and mortality were evaluated up to 24 months after surgery or until death. 


\section{Statistical Analysis}

Main statistical analysis was based on "per protocol" (PP) population, a subset of patients of the intention-to treat (ITT) population fully complying with protocol requirements. Patients treated with reconstruction procedures other than the randomized one or patients undergoing adjuvant pelvic radiotherapy were excluded from the PP population. Therefore, all patients in the PP population were analyzed according to the surgery they actually received.

The sample size of 82 evaluable patients in each treatment arm was calculated according to Horn and Vollandt, ${ }^{21}$ with a type I error rate of 5\% and a power of $90 \%$ for each pair of comparisons. Sample size was then inflated by $15 \%$ resulting in a total of 282 patients. A second safety analysis showed a substantially higher than expected rate of nonevaluable patients (33\%). Therefore, patients' number was further increased $(n=54)$ to a total of 336 .

For primary analysis, composite evacuation scores associated with the 3 reconstruction techniques at 12 months were compared using pairwise, 2-sided, Steel multiple comparison Wilcoxon tests. ${ }^{22}$

For other analyses, overall comparisons across arms were carried out using chi-squared, Kruskal-Wallis, and log-rank tests for categorical, continuous, and time-to-event variables, respectively. If there were significant overall differences, pairwise comparisons were carried out using chi-squared, Mann-Whitney, and log-rank tests for categorical, continuous, and time-to-event variables. ${ }^{23}$

To take into account covariates other than treatment arm, linear mixed effect models, with surgeon as random effect, were used for continuous variables. Continuous data were summarized using median and range, and categorical data using frequency and percentage.

Two-tailed tests with significance level of 0.05 were used for all analyses. As no adjustment for multiple testing other than primary endpoint evaluation was applied, analyses were exploratory and hypothesis generating. All analyses were performed using SAS 9.4 (SAS Institute) and R 3.2.4 (http://www.r-project.org).

\section{Ethics and Safety Surveillance}

It was anticipated that for a certain number of patients, randomized reconstruction would not be feasible due to technical reasons such as length of remaining colon not allowing to safely perform CJP. As patient's safety was the most important factor, any uncertain condition during surgery allowed the surgeon to overrule randomization. In these cases, the surgeon could choose one of the other techniques.

Laparoscopic resection was allowed if the operating surgeon had an experience of $>30$ laparoscopic colorectal resections prior to participation to the trial. Surgery, neoadjuvant, and adjuvant treatments were applied individually, as recommended by local tumor boards, and according to accepted international guidelines. ${ }^{17}$

The SAKK Coordinating Center in Bern supervised the study at all 15 participating surgical sites and provided continuous on-site monitoring. Surgical and other complications were analyzed as anonymized reports being evaluated and submitted yearly to an international "surgical safety panel" composed of surgeons not involved in any way in the trial.

A first interim safety analysis was performed after inclusion of 50 patients in the trial. Decision to stop the study would have been based upon safety concerns, surgical noncompliance and low accrual. Criteria for this decision were specified in the protocol and strictly followed after the results of the interim safety analysis became available. The trial was allowed to continue upon consideration of all 5 conducted interim safety analyses.

The trial, registered with ClinicalTrials.gov, number NCT00238381), was planned and conducted in accordance with the Declaration of Helsinki, Guidelines for Good Clinical Practice, issued by the International Conference on Harmonization and requirements of respective national regulatory authorities. Respective ethics committees of all participating centers approved the trial and written informed consent was obtained from all patients prior to enrolment.

\section{Role of Funding Sources}

The study was supported by Oncosuisse (OCS 01579-082004) and the Swiss State Secretariat for Education, Research and Innovation (SERI). Funders had no influence on study design, data collection, analysis and interpretation, or report writing.

\section{RESULTS}

\section{Patient Population and Data Acquisition}

In the 15 participating hospitals 336 patients were enrolled between November 2005 and May 2014 (Fig. 1). The 4 university centers, 4 referral centers, and 7 peripheral clinics contributed 104 (5-40), 152 (27-44), and 80 (1-33) patients, respectively. One hundred twelve patients were randomized to each treatment arm. Randomized groups did not show any significant difference regarding preoperative characteristics (Table 1). One patient randomized to CJP reconstruction withdrew his consent after surgery had been performed. Moreover, 68 patients ( $\operatorname{arm} \mathrm{CJP}=47$, arm SEA $=12$, arm SCA $=9$ ) were not treated according with the reconstruction procedure they were randomized to. In particular, in 8 patients, rectal cancer could only be removed by abdomino-perineal rectal amputation, and in 5 patients a Hartmann operation was performed. In 1 patient, a colo-anal sleeve anastomosis was constructed below the dentate line. In 3 cases patients randomized for the SCA group underwent SEA because colon was too short and in accord with surgeon's decision. For 10 patients, a preoperatively unplanned adjuvant radiotherapy treatment had to be performed. To exclude any influence of radiation therapy on functional results, these patients were excluded from analysis, according to the protocol. Thus, $\mathrm{PP}$ population included 257 patients $(\mathrm{CJP}=63,56.3 \%$; $\mathrm{SEA}=$ $95,84.8 \%$; $\mathrm{SCA}=99,88.4 \%$ ).

At registration, median age of patients was 68.6 years $(30.9-$ 85.5) in arm CJP, 67.2 years (32.3-88.9) in arm SEA, and 66.3 years (32.3-90.9) in arm SCA. Median BMI was, $24.2 \mathrm{~kg} / \mathrm{m}^{2}$ in arm CJP, $24.3 \mathrm{~kg} / \mathrm{m}^{2}$ in arm SEA and $25.2 \mathrm{~kg} / \mathrm{m}^{2}$ in arm SCA. Patient and tumor characteristics are summarized in Table 1 . Due to a variety of reasons (patient lost for follow-up, too ill, refusing to cooperate, missing answers or unknown background), composite evacuations, and composite incontinence scores were not recorded after $6,12,18$, and 24 months for $23,27,34$, and 34 patients and 23, 24, 32, and 32 patients, respectively.

\section{Clinical and Oncological Outcome}

In 164 of 322 patients $(50,9 \%)$, surgery was performed laparoscopically. Overall morbidity rate was $50.3 \%(162 / 322)$ and included pulmonary, cardiac, renal, gastrointestinal, and thromboembolic complications, as well as bleeding and infections. Surgical site infection rate was $27.3 \%$ including superficial infection only in $7.5 \%$ (24/322), deep incisional infection in $2.8 \%$ (9/322), and abdominal infection in $12.4 \%(40 / 322)$ of patients. Anastomotic leak rate was $12.1 \%$ (39/322). During initial hospital stay, $14.3 \%$ (46/322) of patients needed surgical reintervention. Stomas from 236 patients of the ITT population $(76 \%)$ and 193 of the PP patient population (82\%) were closed within 9 months following TME. Thirty days mortality among all patients was $1.79 \%$ (6/335).

Tumor resection was confirmed to be complete (R0) in $96.6 \%$ (311/322), microscopically incomplete (R1) in $1.9 \%(6 / 322)$, and staged as Rx or unknown in $1.6 \%(5 / 322)$ of patients. In specimens 


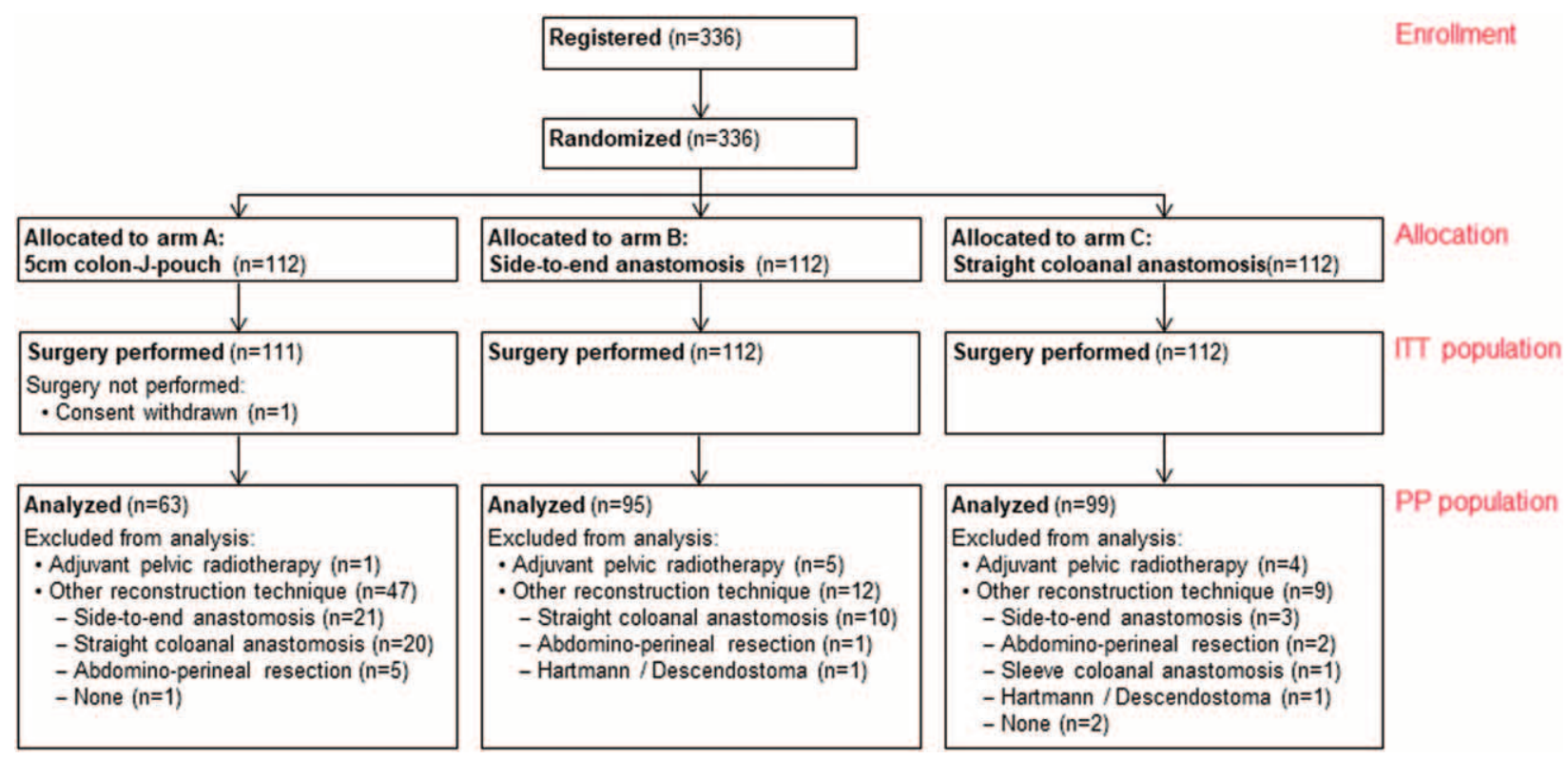

FIGURE 1. Trial profile.

from the 311 completely resected patients, median circumferential resection margin was $12 \mathrm{~mm}(0.5-70 \mathrm{~mm})$, and median distal resection margin was $25 \mathrm{~mm}(2-99 \mathrm{~mm})$. Overall, 34 of $335(10.1 \%)$ patients experienced a relapse (local, distant, or both) within 1 year following surgery, and 59 of $335(17,6 \%)$ within 24 months. Overall survival in the cohort was $94.6 \%$ (95\% confidence interval (CI) 91.6-96.6\%), and $90.6 \%$ (95\% CI 87.0-93.3\%) at 12 and 24 months, respectively.

No statistically significant differences in morbidity, mortality, local or distant metastases, and overall survival were observed among patients included in the different treatment arms (supplementary extended results, Tables 93-104, http://links.lww.com/SLA/ B515).

\section{Evacuation Capacity}

Functional assessments were performed at defined times after TME. $^{8}$ Among the 257 patients in PP populations, composite evacuation scores at $6,12,18$, and 24 months following surgery were available for $160(62.3 \%), 194(75.5 \%), 188(73.2 \%)$, and 179 $(69.6 \%)$ patients. Remaining patients could not be analyzed due to permanent stoma (Hartmann procedure or abdomino-perineal resection), stoma still in place, death, too ill, refusal to cooperate, lost for follow-up or missing data. Composite evacuation scores for PP populations did not show any statistically significant difference among the 3 groups under investigation at any time point considered (Fig. 2).

To strengthen the significance of our data, primary analysis was repeated for the whole ITT population $(n=335)$. Composite evacuation scores at $6,12,18$, and 24 months after surgery were available for 185 (55\%), 232 (69\%), $229(68 \%)$, and $213(64 \%)$ patients, respectively. Again, no significant differences were detectable at any time point (Fig. 2).

Importantly, neoadjuvant treatment did not appear to be associated with unfavorable, higher composite evacuation scores following surgery, at any time point under investigation, irrespective of surgical procedures, and in both PP and ITT populations (supplementary Table 1A-B, http://links.lww.com/ SLA/B515).

\section{Capacity of Continence (Secondary Outcome)}

Among the 257 patients in PP population, composite incontinence scores at $6,12,18$, and 24 months after surgery were available for $160(62.3 \%), 197(76.7 \%), 188(76.7 \%)$, and181 (70.4\%) patients, respectively. Remaining patients could not be analyzed due to permanent stoma (Hartmann procedure or abdomino-perineal resection), stoma still in place, death, too ill and refusal to answer specific questions, lost for follow-up or missing data. Composite incontinence scores for the PP population showed no statistically significant difference among the 3 arms under investigation at any time point, as depicted in Figure 3. Similar observations were made for the ITT population, as well.

Quality of life data will be reported in a dedicated paper (Ribi et al., 2018, in preparation). Nevertheless, remarkably, patients with more severe evacuation problems did not report a significantly worse quality of life, as compared to patients with less severe evacuation problems.

Consistent with evacuation scores (see above), neoadjuvant treatment prior to surgery did not affect composite incontinence scores in PP and ITT populations (supplementary Table 2A-B, http:// links.lww.com/SLA/B515). On the other hand, levels of anastomosis appeared to impact composite incontinence scores at 12, 18, and 24 months in both PP and ITT populations (supplementary Table 3AB, http://links.lww.com/SLA/B515). However, due to high data dispersion, significance threshold was not reached.

\section{DISCUSSION}

Bowel dysfunction represents a major problem following TME. A variety of surgical procedures have been developed to prevent anterior resection syndrome. ${ }^{24,25}$ SCA, CJP, and SEA are the most widely used techniques. Other procedures are less frequently utilized. In particular, transverse coloplasty pouch ${ }^{7}$ has been associated with a higher leak rate, as compared with $\mathrm{JP}^{10}{ }^{10}$ and ileocecal interposition as neorectum is less established, ${ }^{27}$ mainly because of potential risks and absence of functional advantages. ${ }^{11}$

A comprehensive, comparative analysis of functional outcomes associated with SCA, CJP, and SEA is still lacking. 
TABLE 1. Preoperative Patient Characteristics (PP Population)

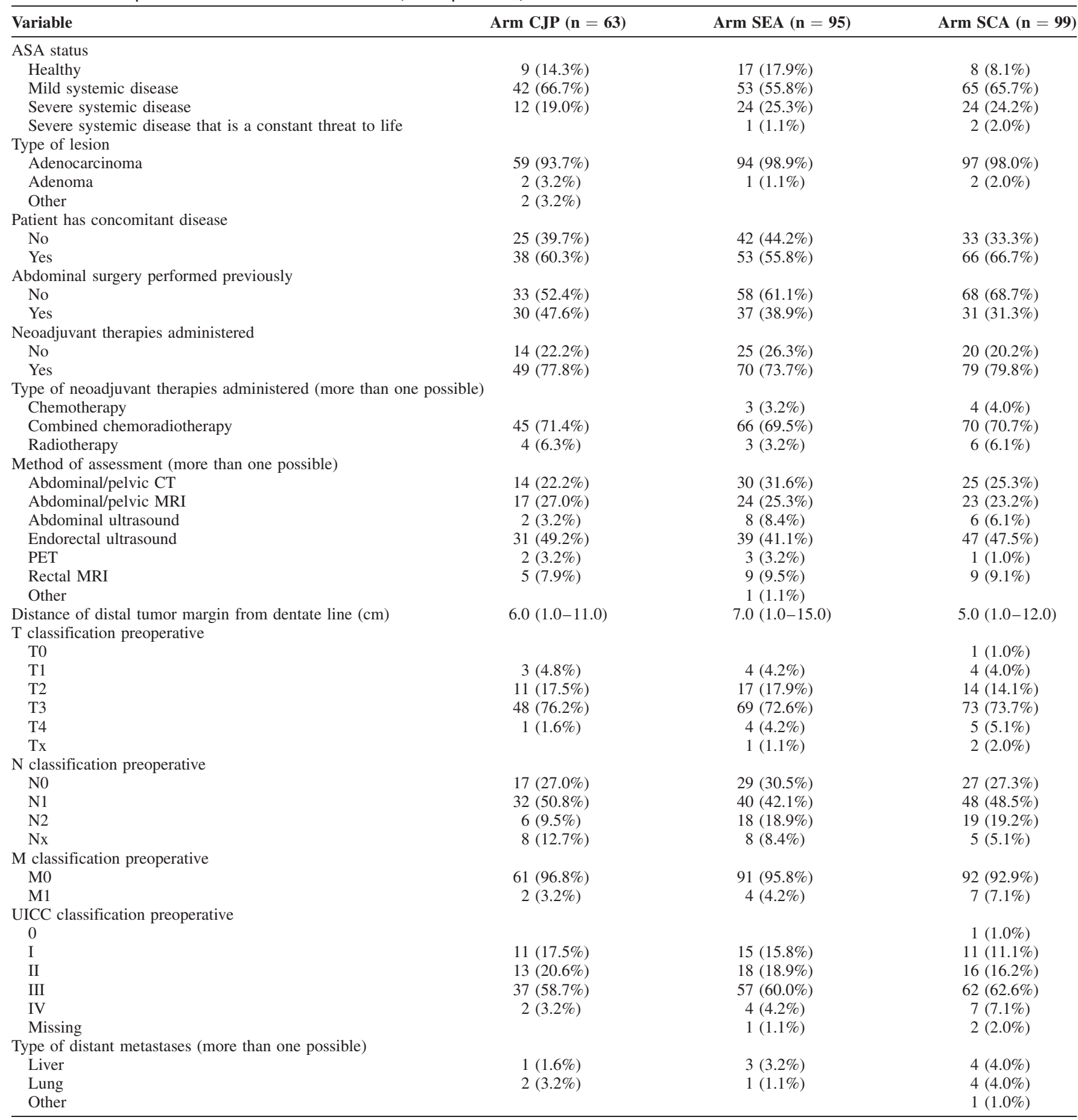

In a prospective, randomized, 2 -arm trial, Hallböök et al ${ }^{12}$ observed an advantageous incontinence score for CJP reconstructed patients over no pouch formation after 2 months, less evident 12 months after surgery. However, in this study only 93 patients were analyzed for functional outcome. Furthermore, pouch was up to $8 \mathrm{~cm}$ long and $10 \%$ of pouch patients needed enemas on regular basis for stool evacuation.
Other studies have compared CJP and SCA in smaller groups of patients $(\mathrm{n} \leq 48) .{ }^{13,14}$ Furthermore, functional outcomes were only evaluated at 3 to 12 months. CJP advantage was only observed in early months after surgery, or was undetectable. ${ }^{13,14}$ A meta-analysis, pooling results from comparative studies revealed a functional advantage for pouch reconstruction up to 1 year following surgery. ${ }^{26}$ A more recent meta-analysis indicates that, CJP, SEA, or transverse 

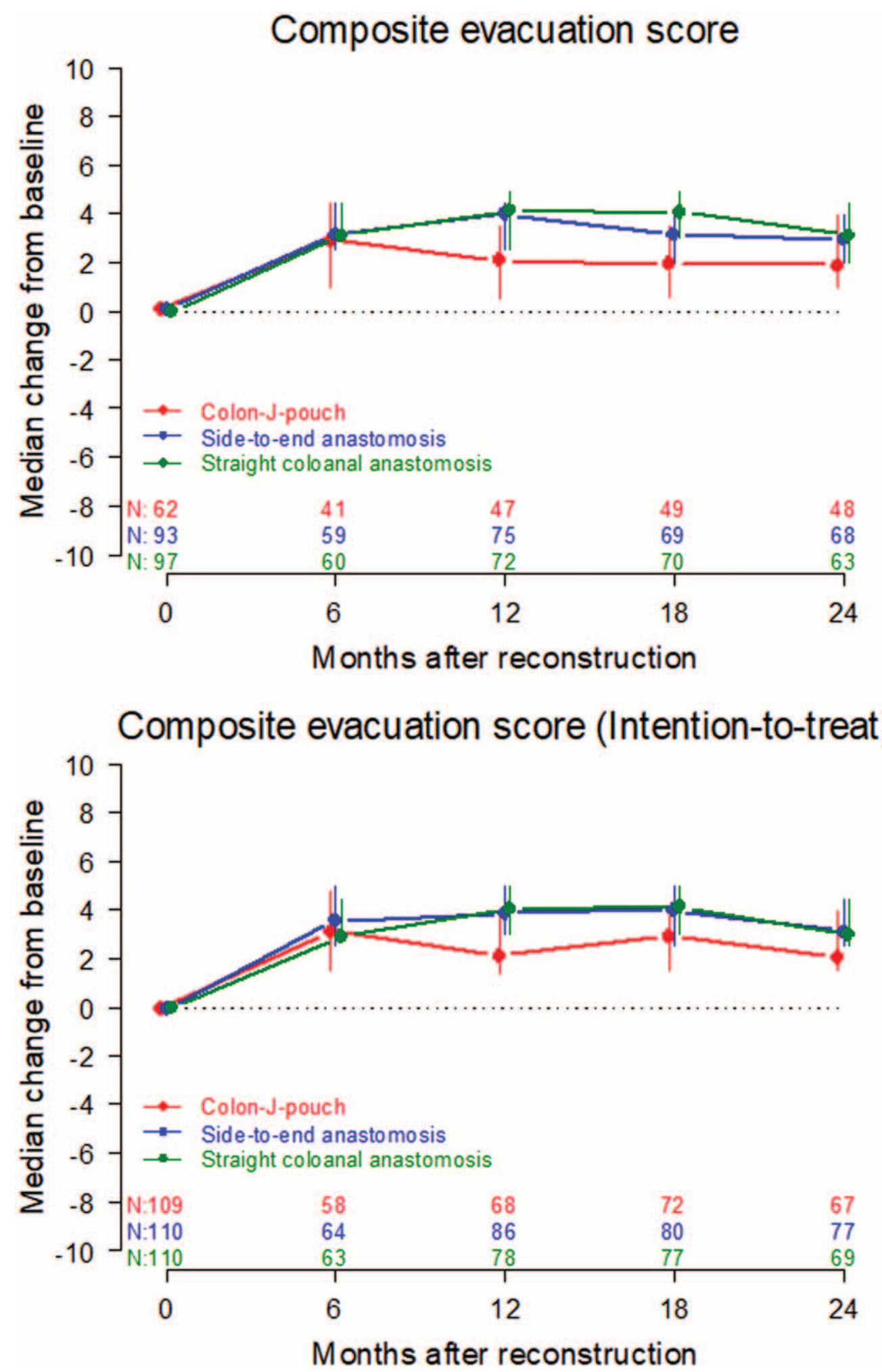

FIGURE 2. Composite evacuation scores. Randomized patients were treated according to the indicated surgical procedures. Evacuation scores were evaluated at the indicated time points. Data are expressed as median changes from baseline, with $95 \%$ confidence limits. Data refer to protocol (PP, upper panel) and intention-to-treat (ITT, lower panel) populations, respectively. 


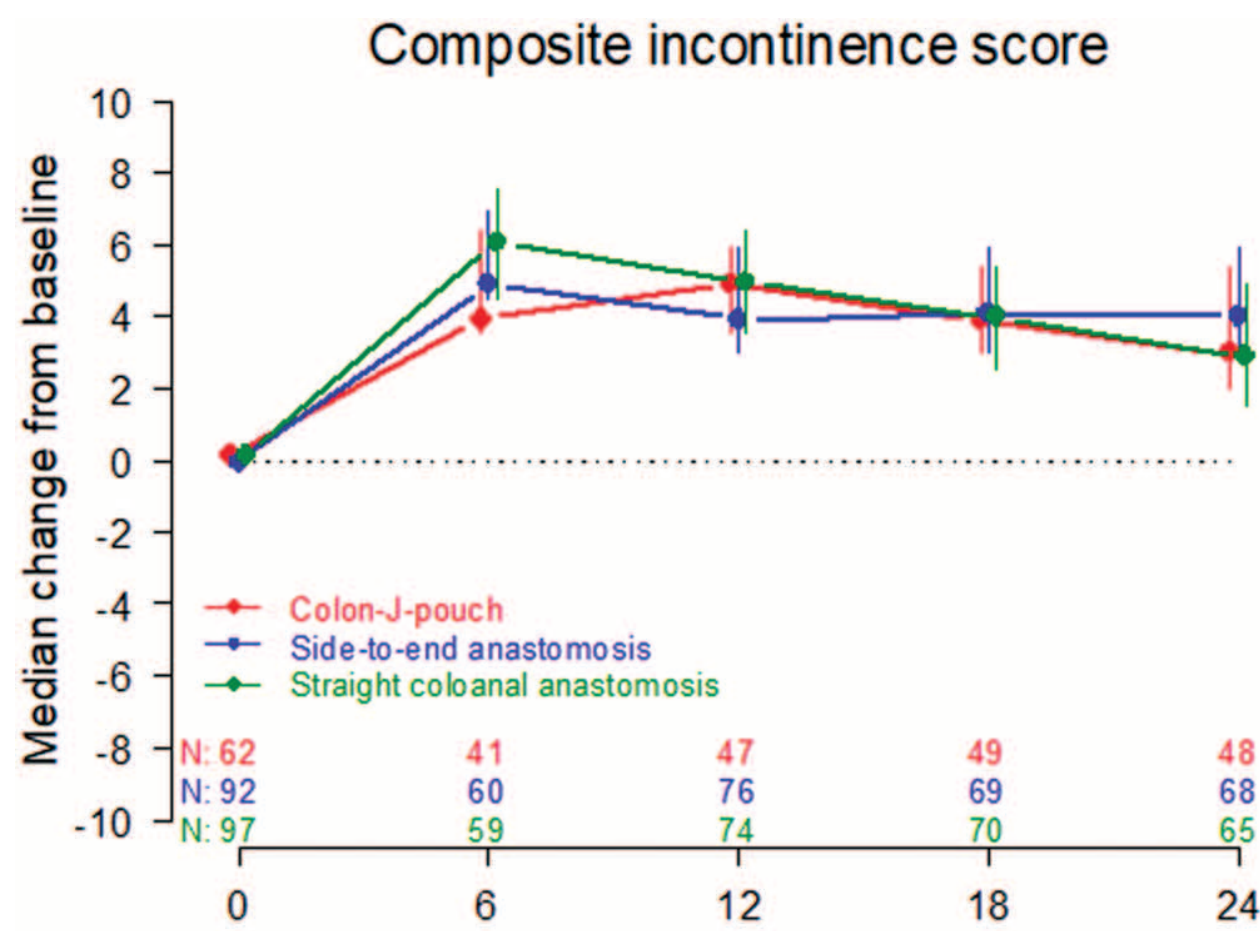

Months after reconstruction

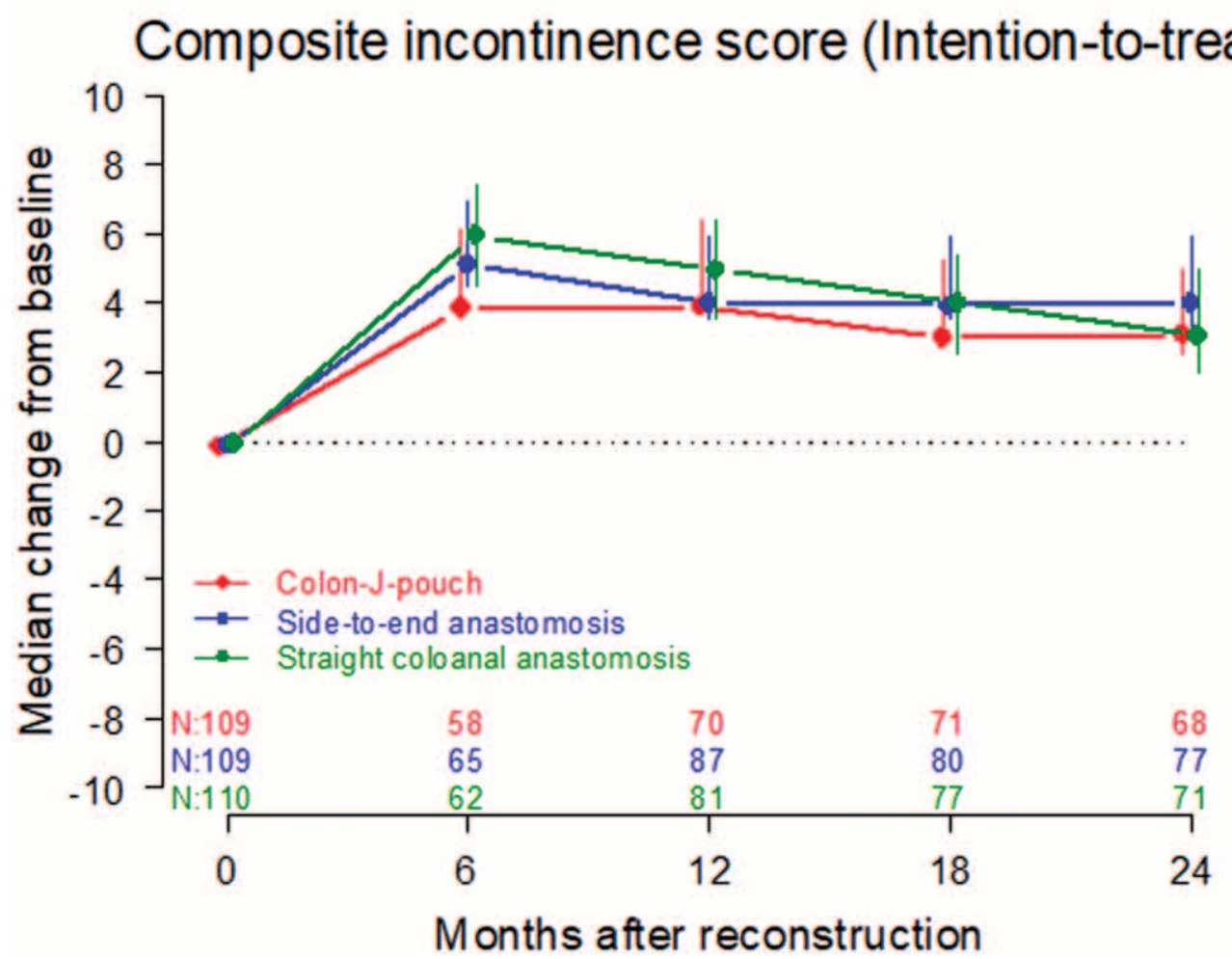

FIGURE 3. Composite incontinence scores. Composite incontinence scores were obtained from randomized patients treated according to the indicated surgical procedures at the indicated time points. Data, expressed as median changes from baseline with 95\% confidence limits, refer to protocol (PP, upper panel) and intention-to-treat (ITT, lower panel) populations, respectively. 
coloplasty reconstruction do show advantages over SCA only within the first year following surgery. ${ }^{27}$ However, nonrandomized studies have also been included in this report.

Markovic et al could not observe improved defecatory function upon CJP, as compared with SEA reconstruction, in a study including 80 patients, at 6,12 , and 24 months. ${ }^{28}$ These results are largely in accordance with a meta-analysis by $\mathrm{Si}$ et $\mathrm{al}^{15}$ and data from a randomized study by Machado et al. ${ }^{16}$

To our knowledge, our clinical trial is the largest prospective, randomized study in the field of rectal cancer surgery, investigating functional outcomes of 2 different pouch reconstruction techniques in comparison with SCA baseline treatment. Altogether, it has involved 15 hospitals, including universities, referral centers, and regional hospitals, each contributing from 1 to 44 rectal resections. Remarkably, despite this variety, results are comparable, in terms of technical and oncological quality, to other published series. ${ }^{1,29-31}$ For instance, anastomotic leakage rate represents a reliable technical surrogate marker for quality control, and Boyce et $\mathrm{al}^{32}$ have reported a leakage rate of $12.9 \%$ following laparoscopic rectal resection with anastomoses below $6 \mathrm{~cm}$. Indeed, a comparable leakage rate (12.1\%) was also observed in our study.

Our data clearly document that, regarding evacuation, formation of neo-rectal pouch does not provide functional advantages, as compared with SCA and SEA, over a 6 to 24 months postoperative observation. Furthermore, the type of reconstruction does not appear to influence continence capacity.

This study also provides additional results of potential clinical relevance. In particular, although neoadjuvant therapy has been suggested to increase the risk of bowel dysfunction following surgery, ${ }^{33-37}$ it did not appear to be associated with unfavorable evacuation or incontinence scores in our patients. However, our findings should be considered cautiously, since numbers of untreated patients, analyzed as controls (Table 1 and extended results, pages $20-21)$, were relatively low $(n=24,28$, and 25 , respectively, for the 3 arms under investigation in the ITT population, and $n=14,25$, and 20 , respectively, for the 3 arms under investigation in the PP population).

While the trial was not designed to investigate incontinence as related to level of anastomoses, nevertheless, our data may suggest a worse long-term continence capacity for patients bearing anastomoses performed close to or at the dentate line. These findings are in line with published reports investigating the functional impact of anastomotic levels following CJP anastomoses. ${ }^{38}$

Limitations of our study should be acknowledged. In particular, an important limitation might be represented by the use of relatively rarely utilized composite evacuation and incontinence scores. ${ }^{20}$ However, currently validated scores, such as the LARS score ${ }^{19}$ were not available at the time of the initiation of this clinical trial. Furthermore, although they are simple and easily applied in an international context, ${ }^{39}$ they largely fail to account for evacuationrelated symptoms frequently observed following TME, including use of medications to evacuate, stool fragmentation, leading to difficulties to empty, digitation to evacuate, feeling of incomplete evacuation, straining to evacuate, and increased time needed to evacuate. In contrast, evaluation of these symptoms is specifically included in the evacuation score utilized in our study, initially developed by Heald's group. ${ }^{20}$ Furthermore, additional frequently observed symptoms, ${ }^{8}$ including warning before passing motion, ability to differentiate gas from feces, and wearing a pad during the day or at night, are considered in the composite incontinence score, but are not addressed in simpler instruments. Therefore, the questionnaire utilized in our study ${ }^{20}$ provides a highly detailed picture of symptoms experienced by patients following TME.
On the other hand, surgeon's human contact to the patients formed the basis to motivate them for participation in a study, where one of the most important surgical steps, reconstruction, was chosen at random. Therefore, only a fraction of all patients treated within participating centers could be included in this study, thereby explaining their low numbers per year and center. Furthermore, unsurprisingly, particularly in patients randomized to the CJP arm, due to safety reasons, surgeons in charge occasionally reconstructed bowel continuity not according to randomized, but rather to technically simpler procedures. This may weaken, to some extent, the level of evidence of our results. However, regarding postoperative assessments and interviews, study design precluded any influence of patient's gratitude for the individual surgeon. Indeed, study nurses acquired data in a centralized, nonclinical and non-hospital, office setting. Furthermore, due to limited number of study nurse staff, observer-related variability was minimal, with low potential biases.

In conclusion, our data indicate that, within the boundaries of the 3 procedures investigated in this study, surgeons in charge may continue to perform reconstruction of intestinal continuity following TME at their technical preference.

\section{REFERENCES}

1. McDonald PJ, Heald RJ. A survey of postoperative function after rectal anastomosis with circular stapling devices. Br J Surg. 1983;70:727-729.

2. Batignani G, Monaci I, Ficari F, et al. What affects continence after anterior resection of the rectum? Dis Colon Rectum. 1991;34:329-335.

3. Lazorthes F, Fages P, Chiotasso P, et al. Resection of the rectum with construction of a colonic reservoir and colo-anal anastomosis for carcinoma of the rectum. Br J Surg. 1986;73:136-138.

4. Parc R, Tiret E, Frileux P, et al. Resection and colo-anal anastomosis with colonic reservoir for rectal carcinoma. Br J Surg. 1986;73:139-141.

5. Baker JW. Low end to side rectosigmoidal anastomosis; description of technic. Arch Surg. 1950;61:143-157.

6. von Flüe M, Harder F. New technique for pouch-anal reconstruction after total mesorectal excision. Dis Colon Rectum. 1994;37:1160-1162.

7. Z'graggen K, Maurer CA, Büchler MW. Transverse coloplasty pouch. A novel neorectal reservoir. Dig Surg. 1999;16:363-366.

8. Fazio VW, Zutshi M, Remzi FH, et al. A randomized multicenter trial to compare long-term functional outcome, quality of life, and complications of surgical procedures for low rectal cancers. Ann Surg. 2007;246:481-486.

9. Parks AG. Transanal technique in low rectal anastomosis. Proc R Soc Med. 1972;65:975-976.

10. Ho YH, Brown S, Heah SM, et al. Comparison of J-pouch and coloplasty pouch for low rectal cancers: a randomized, controlled trial investigating functional results and comparative anastomotic leak rates. Ann Surg. 2002;236:49-55.

11. Rink AD, Haaf F, Knupper N, et al. Prospective randomised trial comparing ileocaecal interposition and colon-J-pouch as rectal replacement after total mesorectal excision. J Colorectal Dis. 2007;22:153-160.

12. Hallböök O, Påhlman L, Krog M, et al. Randomized comparison of straight and colonic J pouch anastomosis after low anterior resection. Ann Surg. 1996;224:58-65.

13. Liang JT, Lai HS, Lee PH, et al. Comparison of functional and surgical outcomes of laparoscopic-assisted colonic J-pouch versus straight reconstruction after total mesorectal excision for lower rectal cancer. Ann Surg Oncol. 2007; 14:1972-1979.

14. Sailer M, Fuchs KH, Fein M, et al. Randomized clinical trial comparing quality of life after straight and pouch coloanal reconstruction. Br J Surg. 2002;89:1108-1117.

15. Si C, Zhang Y, Sun P. Colonic J-pouch versus Baker type for rectal reconstruction after anterior resection of rectal cancer. Scand J Gastroenterol. 2013;48:1428-1435.

16. Machado M, Nygren J, Goldman S, et al. Similar outcome after colonic pouch and side-to-end anastomosis in low anterior resection for rectal cancer: a prospective randomized trial. Ann Surg. 2003;238:214-220.

17. Schmiegel W, Pox C, Reinacher-Schick A, et al. S3 guidelines for colorectal carcinoma: results of an evidence-based consensus conference on February 6/ 7, 2004 and June 8/9, 2007 (for the topics IV, VI and VII). Z Gastroenterol. 2010;48:65-136. 
18. MacFarlane JK, Ryall RD, Heald RJ. Mesorectal excision for rectal cancer. Lancet. 1993;341:457-460.

19. Juul T, Ahlberg M, Biondo S, et al. International validation of the low anterior resection syndrome score. Ann Surg. 2014;259:728-734.

20. Amin AI, Hallbook O, Lee AJ, et al. A $5-\mathrm{cm}$ colonic J pouch colo-anal reconstruction following anterior resection for low rectal cancer results in acceptable evacuation and continence in the long term. Colorectal Dis. 2003;5:33-37.

21. Horn M, Vollandt R. A survey of sample size formulas for pairwise and manyone multiple comparisons in the parametric, nonparametric and binomial case. Biometrical Journal. 2000;42:27-44.

22. Steel RGD. Some rank sum multiple comparison tests. Biometrics. 1961;17:539-552.

23. Lehmann EL. Nonparametrics: Statistical methods based on ranks. SpringerVerlag; 2006.

24. Kakodkar R, Gupta S, Nundy S. Low anterior resection with total mesorectal excision for rectal cancer: functional assessment and factors affecting outcome. Colorectal Dis. 2006;8:650-656.

25. Hamel CT, Metzger J, Curti G, et al. Ileocecal reservoir reconstruction after total mesorectal excision: functional results of the long-term follow-up. Int $J$ Colorectal Dis. 2004;19:574-579.

26. Heriot AG, Tekkis PP, Constantinides V, et al. Meta-analysis of colonic reservoirs versus straight coloanal anastomosis after anterior resection. $\mathrm{Br}$ J Surg. 2006;93:19-32.

27. Hüttner FJ, Tenckhoff S, Jensen K, et al. Meta-analysis of reconstruction techniques after low anterior resection for rectal cancer. $\mathrm{Br} J$ Surg. 2015;102:735-745

28. Marković V, Dimitrijević I, Barišić G, et al. Comparison of functional outcome of colonic J-pouch and latero-terminal anastomosis in low anterior resection for rectal cancer. Srp Arh Celok Lek. 2015;143:158-161.

29. Dutch Snapshot Research Group. Benchmarking recent national practice in rectal cancer treatment with landmark randomized controlled trials. Colorectal Dis. 2017;19:219-231.
30. Hain E, Maggiori L, Manceau G, et al. Oncological impact of anastomotic leakage after laparoscopic mesorectal excision. Br J Surg. 2017;104:288-295.

31. Kiehlmann M, Weber K, Göhl J, et al. The impact of surgical quality on prognosis in patients undergoing rectal carcinoma surgery after preoperative chemoradiation. Int J Colorectal Dis. 2016;31:247-255.

32. Boyce SA, Harris C, Stevenson A, et al. Management of low colorectal anastomotic leakage in the laparoscopic era: more than a decade of experience. Dis Colon Rectum. 2017;60:807-814.

33. Marijnen CA, van de Velde CJ, Putter H, et al. Impact of short-term preoperative radiotherapy on health-related quality of life and sexual functioning in primary rectal cancer: report of a multicenter randomized trial. J Clin Oncol. 2005;23:1847-1858.

34. Parc Y, Zutshi M, Zalinski S, et al. Preoperative radiotherapy is associated with worse functional results after coloanal anastomosis for rectal cancer. Dis Colon Rectum. 2009;52:2004-2014.

35. Stephens RJ, Thompson LC, Quirke P, et al. Impact of short-course preoperative radiotherapy for rectal cancer on patients' quality of life:data from the Medical Research Council CR02/National Cancer Institute of Canada Clinical Trials Group C016 randomized clinical trial. J Clin Oncol. 2010;28:42334239 .

36. Emmertsen KJ, Laurberg S. Rectal Cancer Function Study Group. Impact of bowel dysfunction on quality of life after sphincter-preserving resection for rectal cancer. Br J Surg. 2013;100:1377-1387.

37. Bregendahl S, Emmertsen KJ, Lous J, et al. Bowel dysfunction after low anterior resection with and without neoadjuvant therapy for rectal cancer: a population-based cross-sectional study. Colorectal Dis. 2013;15:1130-1139.

38. Otto S, Kroesen AJ, Hotz HG, et al. Effect of anastomosis level on continence performance and quality of life after colonic J-pouch reconstruction. Dig Dis Sci. 2008;53:14-20.

39. Battersby NJ, Bouliotis G, Emmertsen KJ, et al. Development and external validation of a nomogram and online tool to predict bowel dysfunction following restorative rectal cancer resection: the POLARS score. Gut. 2018;67:688-696. 